DCP-13-03

\title{
Dirac neutrinos from flavor symmetry
}

\author{
Alfredo Aranda, ${ }^{1,2 *}$ Cesar Bonilla,${ }^{3 \dagger}$ S. Morisi,${ }^{4 \ddagger}$ E. Peinado,${ }^{5 \S}$ J. W. F. Valle, ${ }^{3 \uparrow}$ \\ ${ }^{1}$ Facultad de Ciencias - CUICBAS, Universidad de Colima, Colima, Mexico. \\ ${ }^{2}$ Dual C-P Institute of High Energy Physics, Mexico. \\ 3 AHEP Group, Institut de Física Corpuscular - \\ C.S.I.C./Universitat de València, Parc Cientific de Paterna. \\ C/ Catedratico Jose Beltran, 2 E-46980 Paterna (Valencia) - SPAIN \\ 4 Institut für Theoretische Physik und Astrophysik, \\ Universität Würzburg, 97074 Würzburg, Germany \\ 5 INFN, Laboratori Nazionali di Frascati, \\ Via Enrico Fermi 40, I-00044 Frascati, Italy.
}

(Dated: October 15, 2018)

\begin{abstract}
We present a model where Majorana neutrino mass terms are forbidden by the flavor symmetry group $\Delta(27)$. Neutrinos are Dirac fermions and their masses arise in the same way as those of the charged fermions, due to very small Yukawa couplings. The model fits current neutrino oscillation data and correlates the octant of the atmospheric angle $\theta_{23}$ with the magnitude of the lightest neutrino mass, with maximal mixing excluded for any neutrino mass hierarchy.
\end{abstract}

PACS numbers: 14.60.Pq, 11.30.Hv, 12.10.-g, 12.60.Jv

\section{Introduction}

The historic observation of neutrino oscillations [1-4] implies that neutrinos are massive in contrast with the Standard Model (SM) prediction. Incorporating small masses requires an extension of the SM in which neutrinos are generally expected to be of Majorana type, hence violating lepton number symmetry [5] ${ }^{1}$. On the other hand in many schemes, such as for example the socalled seesaw mechanism lepton number violation is expected to account for the observed smallness of neutrino mass relative to that of charged fermions [5]. Yet, so far current neutrino oscillation experiments have been insensitive to the Majorana nature of neutrinos [7, 8] and, despite intense ongoing efforts it has not been confirmed through the

\footnotetext{
*Electronic address:fefo@ucol.mx

$\dagger$ Electronic address:cesar.bonilla@ific.uv.es

$\ddagger$ Electronic address:stefano.morisi@gmail.com

$\S$ Electronic address:eduardo.peinado@lnf.infn.it

๑Electronic address:valle@ific.uv.es

${ }^{1}$ Recently it has been claimed that one can find models where lepton number is violated by four units, $\Delta L=4$, even if neutrinos are of the Dirac type [6].
}

observation of lepton number violation processes such as neutrinoless double beta decay $(0 \nu \beta \beta)$ 9. Hence neutrinos could very well be Dirac fermions [10. In short, the status of lepton and baryon number symmetries remains as one of the deepest unsolved mysteries of nature [11. An equally puzzling challenge is associated to the origin of the peculiar flavor pattern of mixing angles indicated by global fits of neutrino oscillation experiments 12 .

Here we suggest a possible interconnection between these puzzles, namely, that lepton number conservation can be an accidental consequence of the flavor symmetry that accounts for the neutrino mixing pattern.

Over the last decade non-Abelian discrete groups have been widely used as family symmetries because of their potential in restricting neutrino mixing patterns [13, 14]. As examples we mention the successful models based on the $A_{4}$ group predicting $\theta_{23}=\pi / 4$ and $\theta_{13}=0$ [15, 16]. However the recent discovery of a large reactor angle, $\theta_{13}>0$ [2-4], and a possible hint in favor of non-maximal atmospheric mixing present in recent oscillation fits $\theta_{23}$ [12 suggests the need for generalizing these models [17 and/or seeking for alternative schemes based upon different fla- 
vor symmetries [18].

Here we present a flavor model for leptons using the non-Abelian group $\Delta(27)$ [19 22 that is able to provide automatic lepton number conservation in a way consistent with current global fits of neutrino oscillation data [12]. Recently other non-Abelian flavor symmetries have been used for pure Dirac neutrinos, see for instance [10, 23 25], however Majorana mass terms are forbidden by means of extra Abelian symmetries. Here we focus on the possibility that Majorana mass terms are not allowed from the flavor symmetry without requiring any extra additional Abelian symmetry. We note that since neutrinos are Dirac fermions $0 \nu \beta \beta$ is exactly forbidden. In addition the model gives a correlation between neutrino oscillation parameters that excludes the maximal $\theta_{23}$ value.

\section{Preliminaries}

In order to generate Dirac neutrino mass terms we introduce singlet "right handed" $(\mathrm{RH})$ neutrinos transforming under the flavor symmetry group $\mathcal{G}_{F}$ in such a way that their tensor product does not contain the trivial element of $\mathcal{G}_{F}$. This means that, even though lepton number conservation is not necessarily required a priori, Majorana mass terms are forbidden in the Lagrangian as a result of the flavor symmetry $\mathcal{G}_{F}$.

Although this may be achieved by using an Abelian symmetry $\mathbb{Z}_{N} \forall N \geq 3$ our focus relies on simple non-Abelian flavor symmetry groups. We assume that RH-neutrinos $\left(N_{R}\right)$ transform as a 3-dimensional irreducible representation (irrep) of $\mathcal{G}_{F}$. Hence if $N_{R}$ transforms as 3-dimensional irrep (3) under $\mathcal{G}_{F}$, one finds that the non-Abelian symmetries which forbid a term like $N_{R}^{T} N_{R}$ are

- $\Delta\left(3 N^{2}\right)$ for $N \geq 3$ : these groups contain nine singlets and $\left(N^{2}-3\right) / 3$ triplets for $N=3 \mathbb{Z}$. Otherwise, for $N \neq 3 \mathbb{Z}$, they have three singlets and $\left(N^{2}-1\right) / 3$ triplets.

- $\Sigma\left(3 N^{3}\right)$ for $N \geq 3$ : the set of groups with $N\left(N^{2}+8\right) / 3$ conjugacy classes, $3 N$ singlets and $N\left(N^{2}-1\right) / 3$ triplets.

\begin{tabular}{|c|c|c|c|c|c|c|}
\hline & $\bar{L}$ & $\ell_{1 R}$ & $\ell_{2 R}$ & $\ell_{3 R}$ & $N_{R}$ & $H$ \\
\hline$S U(2)_{L}$ & $\mathbf{2}$ & $\mathbf{1}$ & $\mathbf{1}$ & $\mathbf{1}$ & $\mathbf{1}$ & $\mathbf{2}$ \\
\hline$\Delta(27)$ & $\mathbf{3}$ & $\mathbf{1}$ & $\mathbf{1}^{\prime}$ & $\mathbf{1}^{\prime \prime}$ & $\mathbf{3}$ & $\mathbf{3}^{\prime}$ \\
\hline
\end{tabular}

Table I: Matter assignments of the model.

- $T_{N}$ for the $N$ values given in [26] these groups have 3 singlets and $(N-1) / 3$ three-dimensional irreducible representations.

- $Z_{9} \rtimes Z_{3}$.

In fact the mass term $N_{R}^{T} N_{R}$ is forbidden because the tensor product $\mathbf{3}_{i} \otimes \mathbf{3}_{i}$ (where $i=$ $1, \ldots, n_{d}$ and $n_{d}=\left(N^{2}-3\right) / 3$ for $\Delta\left(3 N^{2}\right)$ and $n_{d}=N\left(N^{2}-1\right) / 3$ for $\left.\Sigma\left(3 N^{3}\right)\right)$ does not contain a trivial 1-dimensional irrep $1^{0}[26,27]$.

\section{The model}

Searching for the the smallest realistic flavor symmetry group of the above class, i.e. used in the context of forbidding Majorana mass terms, we find that ${ }^{2}$ it is $\Delta(27)$. The $\mathrm{SU}(3)_{\mathrm{c}} \otimes \mathrm{SU}(2)_{\mathrm{L}} \otimes \mathrm{U}(1)_{\mathrm{Y}} \otimes \Delta(27)$ multiplet assignment is given in Table $\mathrm{I},{ }^{3}$. where we have extended the SM by adding three righthanded neutrinos and two Higgs doublets apart from that of the Standard Model. The most general invariant Lagrangian for leptons is written as

$$
\mathcal{L}_{\ell}=\sum_{i=1}^{3} Y_{i}^{\ell} \bar{L} \ell_{i R} H+Y^{\nu} \bar{L} N_{R} \tilde{H}+\text { h.c. },
$$

where we use the compact notation $H=$ $\left(H_{1}, H_{2}, H_{3}\right)$ and $\tilde{H}=\left(\tilde{H}_{1}, \tilde{H}_{2}, \tilde{H}_{3}\right)$ with $\tilde{H}_{i} \equiv i \sigma_{2} H^{*}$. After electroweak symmetry breaking one gets the following patterns for the neutrino and charged lepton mass matri-

${ }^{2} T_{7}$ has the desired product and has indeed been used as a successful flavor symmetry, however not in the context of Dirac neutrinos 28 31.

3 We denote, by convenience, $\mathbf{1} \equiv \mathbf{1}_{(0,0)}, \mathbf{1}^{\prime} \equiv \mathbf{1}_{(1,0)}$, $\mathbf{1}^{\prime \prime} \equiv \mathbf{1}_{(2,0)}, \mathbf{3} \equiv \mathbf{3}_{(0,1)}$ and $\mathbf{3}^{\prime} \equiv \mathbf{3}_{(0,2)}$, where the index notation is that used in 26, 27. 
ces:

$$
\begin{aligned}
M_{\nu}= & {\left[\begin{array}{lll}
a v_{1} & b v_{3} & c v_{2} \\
c v_{3} & a v_{2} & b v_{1} \\
b v_{2} & c v_{1} & a v_{3}
\end{array}\right] } \\
M_{\ell}= & {\left[\begin{array}{ccc}
Y_{1}^{\ell} v_{1} & Y_{2}^{\ell} v_{1} & Y_{3}^{\ell} v_{1} \\
Y_{1}^{\ell} v_{2} & \omega Y_{2}^{\ell} v_{2} & \omega^{2} Y_{3}^{\ell} v_{2} \\
Y_{1}^{\ell} v_{3} & \omega^{2} Y_{2}^{\ell} v_{3} & \omega Y_{3}^{\ell} v_{3}
\end{array}\right] }
\end{aligned}
$$

where $v_{i}$ are Higgs scalar vacuum expectation values (vevs), $\langle H\rangle=\left(\left\langle H_{1}\right\rangle,\left\langle H_{2}\right\rangle,\left\langle H_{3}\right\rangle\right)=$ $\left(v_{1}, v_{2}, v_{3}\right)$. The parameters $\left\{a, b, c, Y_{i}\right\}$ are real if $\mathrm{CP}$ invariance is assumed where the $\mathrm{CP}$ transformation is properly defined in [3235]. One sees that in such minimal scenario the smallness of neutrino masses w.r.t. those of the charged leptons must arise due to very small Yukawa couplings ${ }^{4}$. The structure of $M_{\ell}$ and $M_{\nu}$ are well known in the literature 16, 19 and the alignment $\langle H\rangle=v(1,1,1)$ turns out to be natural in $\Delta(27)$ [19, 21].

In such a case $M_{\ell}$ can be written as $M_{\ell}=$ $U_{\omega} \hat{Y}$ where $\hat{Y}=\operatorname{diag}\left(Y_{1}, Y_{2}, Y_{3}\right)$ and

$$
U_{\omega}=\frac{1}{\sqrt{3}}\left[\begin{array}{ccc}
1 & 1 & 1 \\
1 & \omega & \omega^{2} \\
1 & \omega^{2} & \omega
\end{array}\right]
$$

is the so-called "magic" matrix. However, given the structure of the neutrino mass matrix $M_{\nu}$, the previous alignment $\langle H\rangle=$ $v(1,1,1)$ cannot be assumed since then $U_{\omega}$ diagonalizes both $M_{\nu} M_{\nu}^{\dagger}$ and $M_{\ell} M_{\ell}^{\dagger}$. This results in a trivial lepton mixing matrix

$$
U=U_{\ell}^{\dagger} U_{\nu}=U_{\omega}^{\dagger} U_{\omega}=\mathbb{I}
$$

Moreover, when $v_{1}=v_{2}=v_{3}=v$ and the couplings $a, b$ and $c$ are real the resulting neutrino masses are also not suitable to account for current neutrino oscillation data.

All of this can be avoided by deviating from the simplest vev alignment, i.e. we can fit the neutrino squared mass differences, as well as induce large lepton mixing angles by

4 Suppressed Yukawa coefficients can arise in extra dimension schemes, i.e. [24], as well as supersymmetric schemes, see for instance 23 . assuming that the vev alignment is generalized to

$$
\langle H\rangle=\hat{v}\left(1+\epsilon_{1}, 1+\epsilon_{2}, 1\right)^{T},
$$

where $|\langle H\rangle|^{2}=v^{2}=(246 \mathrm{GeV})^{2}$. The above vev configuration is a solution of the minimization conditions of the scalar potential provided it softly breaks the flavor symmetry, the deviation parameters $\epsilon_{1,2}$ being then associated to this soft breaking.

Taking into account Eq. (5) the mass matrices for the lepton sector are now given by

$$
\begin{aligned}
& M_{\nu}=\hat{v}\left[\begin{array}{ccc}
a\left(1+\epsilon_{1}\right) & b & c\left(1+\epsilon_{2}\right) \\
c & a\left(1+\epsilon_{2}\right) & b\left(1+\epsilon_{1}\right) \\
b\left(1+\epsilon_{2}\right) & c\left(1+\epsilon_{1}\right) & a
\end{array}\right] \\
& M_{\ell}=\hat{v}\left[\begin{array}{ccc}
Y_{1}^{\ell}\left(1+\epsilon_{1}\right) & Y_{2}^{\ell}\left(1+\epsilon_{1}\right) & Y_{3}^{\ell}\left(1+\epsilon_{1}\right) \\
Y_{1}^{\ell}\left(1+\epsilon_{2}\right) & \omega Y_{2}^{\ell}\left(1+\epsilon_{2}\right) & \omega^{2} Y_{3}^{\ell}\left(1+\epsilon_{2}\right) \\
Y_{1}^{\ell} & \omega^{2} Y_{2}^{\ell} & \omega Y_{3}^{\ell}
\end{array}\right] .
\end{aligned}
$$

Note that an immediate consequence of the generalized vev alignment is that the $U_{\omega}$ no longer diagonalizes the neutrino mass matrix nor that of the charged leptons, and therefore, as desired, the lepton mixing matrix is now non-trivial,

$$
U=U_{\ell}^{\dagger} U_{\nu} \neq \mathbb{I}
$$

Furthermore one can indeed fit all neutrino observables as we now show.

\section{Results}

Here we consider deviations of the alignment $v(1,1,1)$ of the order $\mathcal{O}\left(\lambda_{C}\right)$ where $\lambda_{C} \sim 0.2$ is the Cabibbo angle. More precisely, using Eqs. (5) and (6) we have scanned over values for the small parameters $\epsilon_{1,2}$ within the range $\left|\epsilon_{1,2}\right| \leq 0.3$ and selected those solutions which satisfy the global fits for the mixing angles at $3 \sigma$ [12

$$
\begin{gathered}
0.017<\sin ^{2} \theta_{13}<0.033 \\
0.36(0.37)<\sin ^{2} \theta_{23}<0.68(0.67) \mathrm{NH}(\mathrm{IH}) \\
0.27<\sin ^{2} \theta_{12}<0.37
\end{gathered}
$$

as well as the neutrino squared mass differences

$$
\begin{aligned}
\Delta m_{21}^{2} & =(7.12-8.20) \times 10^{-5} \mathrm{eV}^{2} \\
\left|\Delta m_{31}^{2}\right| & =\left\{\begin{array}{ll}
(2.31-2.74) & \text { for } \mathrm{NH} \\
(0.21-2.64) & \text { for } \mathrm{IH}
\end{array}\right\} \times 10^{-3} \mathrm{eV}^{2} .
\end{aligned}
$$




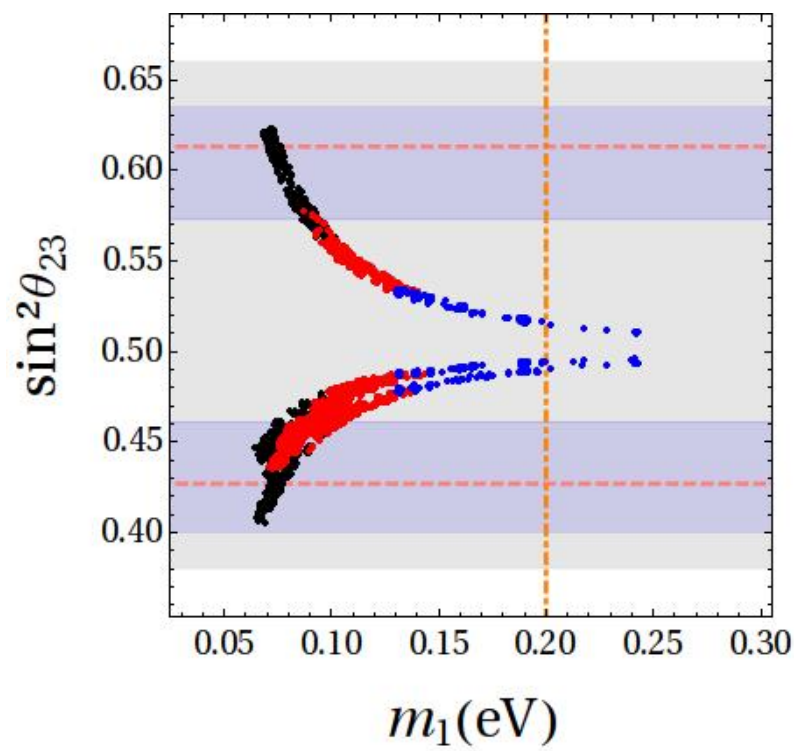

Figure 1: Correlation between the atmospheric angle and the lightest neutrino mass for the NH case. The horizontal dotted lines represent the best fit values, the (dark) blue and (light) gray horizontal bands are the $1 \sigma$ and $2 \sigma$ allowed ranges, respectively. The blue (light gray), red (gray) and black points are model expectations corresponding to vev deviations of $10 \%$, $20 \%$ and $30 \%$ respectively (see text for more details). The vertical dot-dashed line indicates KATRIN's sensitivity 36.

We have found a correlation between the atmospheric angle and the lightest neutrino mass for both the normal mass hierarchy $(\mathrm{NH})$ and the inverted mass hierarchy (IH) cases. This is shown in Figures 1 and 2 for the $\mathrm{NH}$ and $\mathrm{IH}$ cases, respectively. In both figures the dotted horizontal lines represent the best fit values, while the (dark) blue and (light) gray horizontal bands are the $1 \sigma$ and $2 \sigma$ bands obtained in Ref. [12, respectively. For the NH case the global oscillation fit finds also a local minimum in the first octant of $\theta_{23}$ [12].

In order to explore the sensitivity of the observables with respect to the values of the vev deviation parameters, $\epsilon_{1,2}$, we consider the following cases, $\left|\epsilon_{1,2}\right| \lesssim 0.1,\left|\epsilon_{1,2}\right| \lesssim 0.2$ and $\left|\epsilon_{1,2}\right| \lesssim 0.3$ where each one represents deviations of $10 \%, 20 \%$ and $30 \%$ respectively. As we mentioned above, the free parameters

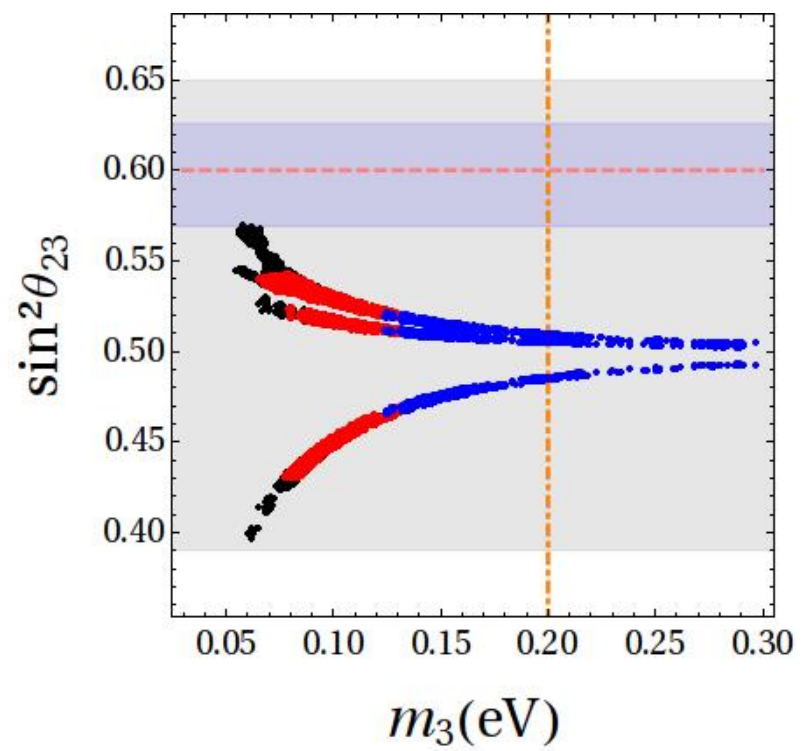

Figure 2: Same as Figure 1 for the IH case. Note that in this case a $30 \%$ vev deviation is not enough to reach the best fit value of $\theta_{23}$.

$\epsilon_{1,2}$ are associated to the $\Delta(27)$-soft breaking terms in the scalar potential and they are allowed to deviate at most at the order of the Cabibbo angle, $\epsilon_{1,2} \sim \mathcal{O}\left(\lambda_{C}\right)$.

The solutions in blue (light gray) correspond to deviations up to $10 \%$, those in red (gray) up to $20 \%$ and those in black up to $30 \%$. Figure 2 for the IH case shows that a $30 \%$ vev deviation is not enough to reach the best fit value for $\theta_{23}$, so that larger deviations would be required in order to accomplish it.

In the near future the KATRIN experiment could discover a neutrino mass in the degenerate region, going from $m_{\beta} \sim 0.3 \mathrm{eV}$ at $3 \sigma$ significance to $m_{\beta}=0.35 \mathrm{eV}$ at $5 \sigma$ significance [36]. If a neutrino mass is not seen in tritium $\beta$ decays this will set an upper bound of $0.2 \mathrm{eV}$ for neutrino mass and such a bound is depicted in each figure with the dot-dashed vertical line.

It is important to note that the atmospheric angle deviates significantly from the maximal value as the vev deviations increase.

Before concluding we mention that the model leads to contributions to flavor changing neutral current (FCNC) processes in the lepton sector, such as $\mu \rightarrow e \gamma$. However, we have checked a few representative points with normal neutrino mass hierarchy, and found that there is sufficient freedom in parameter 
space to satisfy the current MEG bound for such a process [37]. Indeed, Table II gives the expected $\mu \rightarrow e \gamma$ branching ratios such points are all consistent with current bounds. Considering that these points are located in different parameter regions, we believe that a detailed analysis will give similar results, though a complete study is beyond the scope of this paper and will be considered elsewere. Note that the model does not lead to FCNC in the quark sector as its symmetry affects only the lepton sector. A model upgrading the flavour symmetry to both sectors is being developed and will be presented in a future publication, including a detailed phenomenological study.

\begin{tabular}{|c|c|c|c|}
\hline Cases & $\mathrm{Br}^{\text {th }}(\mu \rightarrow e \gamma)$ & $m_{\nu_{1}}(\mathrm{eV})$ & $\sin ^{2} \theta_{23}$ \\
\hline i) & $1.98 \times 10^{-14}$ & 0.2399 & 0.4956 \\
\hline ii) & $1.74 \times 10^{-14}$ & 0.0930 & 0.4615 \\
\hline iii $)$ & $1.65 \times 10^{-14}$ & 0.0762 & 0.6107 \\
\hline
\end{tabular}

Table II: Theoretical branching ratios for the process $\mu \rightarrow e \gamma$ for three different cases corresponding to three different sets of $\left(\epsilon_{1}, \epsilon_{2}\right), m_{\nu_{1}}$, and $\sin ^{2} \theta_{23}$.

\section{Summary}

We have presented a model based on $\Delta(27)$ flavor symmetry. We showed that having $\mathrm{RH}$ neutrinos and LH leptons transforming as 3 dimensional irreps under $\Delta(27)$ forbids Majorana mass terms so that neutrinos are naturally Dirac-type, just as all other Standard Model fermions [10]. There is accidental lepton number conservation in the model caused by gauge symmetry, as in the SM, and it is present before and after EWSB. Furthermore, due to the particle content of the model, we find that all higher order Weinberg-type operators $\operatorname{LHLH}\left(H^{\dagger} H\right)^{n}$ for $n=0,1,2, \ldots$ that might yield a Majorana mass are not allowed by the symmetry $\Delta(27)$ and there are neither scalar singlets nor triplets to realize any diagram (operator) in 38 40]. This scenario is able to fit the current data in the lepton sector and establishes a correlation between the octant of the atmospheric angle $\theta_{23}$ and the magnitude of the lightest neutrino mass eigenvalue which may be probed by coming experiments.

\section{Acknowledgments}

We would like to thank Luis Lavoura and Christoph Luhn for comments on the manuscript. This work was supported by MINECO grants FPA2011-22975, MULTIDARK Consolider CSD2009-00064, by Prometeo/2009/091 (Generalitat Valenciana), by EU ITN UNILHC PITN-GA-2009-237920. A.A thanks support from CONACYT and PROMEP. S.M. thanks DFG grant WI 2639/4-1. C.B. thanks support from EPLANET.

[1] A. McDonald, Talk at XIV International Workshop on Neutrino Telescopes, Venice, March, 2011.

[2] DAYA-BAY Collaboration, F. An et al., Phys.Rev.Lett. 108, 171803 (2012), [1203.1669].

[3] RENO collaboration, J. Ahn et al., 1204.0626 .

[4] T2K Collaboration, K. Abe et al., Phys.Rev.Lett. 107, $041801 \quad$ (2011), [1106.2822].

[5] J. Schechter and J. W. F. Valle, Phys. Rev. D22, 2227 (1980).

[6] J. Heeck and W. Rodejohann, 1306.0580.

[7] J. Schechter and J. W. F. Valle, Phys. Rev. D25, 2951 (1982).

[8] M. Duerr, M. Lindner and A. Merle, JHEP 1106, 091 (2011), [1105.0901].

[9] A. Barabash, 1101.4502, 75 years of double beta decay: yesterday, today and tomorrow.

[10] N. Memenga, W. Rodejohann and H. Zhang, Phys.Rev. D87, 053021 (2013), [1301.2963].

[11] S. Weinberg, Phys. Rev. D22, 1694 (1980).

[12] D. Forero, M. Tortola and J. W. F. Valle, Phys.Rev. D86, 073012 (2012), arXiv:1205.4018].

[13] S. Morisi and J. Valle, Fortschritte der Physik-Progress of Physics 61, 466 (2013).

[14] M. Hirsch et al., 1201.5525.

[15] K. S. Babu, E. Ma and J. W. F. Valle, Phys. 
Lett. B552, 207 (2003), hep-ph/0206292.

[16] G. Altarelli and F. Feruglio, Nucl. Phys. B720, 64 (2005), hep-ph/0504165.

[17] S. Morisi, D. Forero, J. Romao and J. Valle, 1305.6774 .

[18] G.-J. Ding, S. Morisi and J. Valle, Phys.Rev. D87,053013 (2013), [1211.6506].

[19] E. Ma, Mod.Phys.Lett. A21, 1917 (2006), hep-ph/0607056.

[20] I. de Medeiros Varzielas, S. King and G. Ross, Phys.Lett. B648, 201 (2007), hep-ph/0607045.

[21] E. Ma, Phys.Lett. B660, 505 (2008), [0709.0507].

[22] S. Morisi, E. Peinado and A. Vicente, 1212.4145 .

[23] M.-C. Chen, M. Ratz, C. Staudt and P. K. Vaudrevange, Nucl.Phys. B866, 157 (2013), [1206.5375].

[24] G.-J. Ding and Y.-L. Zhou, 1304.2645.

[25] M. Holthausen and K. S. Lim, 1306.4356.

[26] H. Ishimori et al., Prog. Theor. Phys. Suppl. 183, 1 (2010), [1003.3552].

[27] C. Luhn, S. Nasri and P. Ramond, J.Math.Phys. 48, 073501 (2007), hepth/0701188].

[28] C. Hagedorn, M. A. Schmidt and A. Y. Smirnov, Phys.Rev. D79, 036002 (2009), [0811.2955].
[29] Q.-H. Cao, S. Khalil, E. Ma and H. Okada, Phys.Rev.Lett. 106, 131801 (2011), [1009.5415].

[30] K. M. Parattu and A. Wingerter, Phys.Rev. D84, 013011 (2011), [1012.2842].

[31] C. Luhn, K. M. Parattu and A. Wingerter, JHEP 1212, 096 (2012), [1210.1197].

[32] G. Branco, J. Gerard and W. Grimus, Phys.Lett. B136, 383 (1984).

[33] P. Ferreira, W. Grimus, L. Lavoura and P. Ludl, JHEP 1209, 128 (2012), [1206.7072].

[34] M. Holthausen, M. Lindner and M. A. Schmidt, JHEP 1304, 122 (2013), [1211.6953].

[35] C. Nishi, Phys. Rev. D 88, 033010 (2013), [1306.0877].

[36] L. B. KATRIN Collaboration, hep$\mathrm{ex} / 0309007$.

[37] MEG Collaboration, J. Adam et al., 1303.0754.

[38] E. Ma, Phys. Rev. Lett. 81, 1171 (1998), [hep-ph/9805219].

[39] F. Bonnet, D. Hernandez, T. Ota and W. Winter, JHEP 10, 076 (2009), [0907.3143].

[40] F. Bonnet, M. Hirsch, T. Ota and W. Winter, JHEP 1207, 153 (2012), [1204.5862]. 